\title{
Cloning and purification of functionally active Fas ligand interfering protein (FIP) expressed in Escherichia coli
}

\author{
Pawel Wisniewski, Adam Master and Bozena Kaminska ${ }^{\bowtie}$ \\ Laboratory of Transcription Regulation, Department of Cell Biology, \\ The Nencki Institute of Experimental Biology, Warszawa, Poland
}

Received: 05 October, 2007; revised: 04 December, 2007; accepted: 04 January, 2007

available on-line: 18 January, 2008

\begin{abstract}
This report presents purification and characterization of the extracellular domain of rat Fas protein, called FIP (FasL interfering protein), expressed as inclusion bodies in Escherichia coli. FIP was extracted from the inclusion bodies, solubilized with $8 \mathrm{M}$ urea, purified by a single-step immobilized metal ion $\left(\mathrm{Ni}^{2+}\right)$ affinity chromatography and refolded. SDS/PAGE and mass spectrometry analysis of the purified protein verified its purity. Fluorescence spectrum analysis showed that the refolding procedure caused structural changes which presumably might have led to oligomerization. The purified FIP has biological activities: it binds specifically soluble Fas ligand and protects human Jurkat lymphocytes against FasL-dependent apoptosis. This efficient procedure of FIP expression in $E$. coli and renaturation may be useful for production of therapeutically important proteins.
\end{abstract}

Keywords: recombinant protein purification, Fas, Fas ligand, interfering molecules, apoptosis

\section{INTRODUCTION}

The Fas ligand (CD95 ligand) is a 40-kDa type II membrane protein belonging to the tumour necrosis factor (TNF) $\alpha$ family of proteins (Smith et al., 1994; Nagata, 1997). This family consists of trimeric ligands that induce defined cellular responses upon binding to their respective receptors. Fas and other members of the TNF receptor superfamily are type I membrane proteins. They are characterized by the presence of cysteine-rich motifs conferring an elongated structure on their extracellular domains (Nagata, 1997). The interaction of Fas with FasL results in activation of signalling pathways which initiate apoptosis (Scaffidi et al., 1998; Krammer, 2000; Curtin \& Cotter, 2003). Activation-induced cell death and Fas-FasL interactions have been shown to play significant roles in immune system homeostasis (Green, 2003) and apoptosis of anti-tumour immune effector cells (Ryan et al., 2005). The Fas/FasL signalling components are expressed in the central nervous system and their expression strongly increases in neurodegenerative diseases (Ethel \& Buhler, 2003; Choi \& Benveniste, 2004). In addition to its pro-apoptotic functions, accumulating evidence demonstrates that FasL can activate numerous non-apoptotic signalling pathways (Wajant et al., 2003), and that activation of these pathways can result in increased tumorigenicity and metastasis (Barnhart et al., 2004). Thus, antagonizing FasL activity may be beneficial in many pathologic conditions.

A promising way to intervene with biological processes is through the control of protein-protein interactions by means of interfering molecules that modulate the formation of protein-protein complexes. Fas-Fc, which contains the extracellular domain of recombinant human Fas (aa 1-154) fused to the Fc domain of human IgG1 and antagonizes the activa-

\footnotetext{
¿Corresponding author: Bozena Kaminska, Department of Cell Biology, The Nencki Institute of Experimental Biology, Pasteur 3, 02-093 Warszawa, Poland; fax: (48 22) 822 5342; e-mail: bozenakk@nencki.gov.pl

Abbreviations: DMF, dimethylformamide; FasL, Fas ligand; FIP, FasL interfering protein; IPTG, isopropyl- $\beta$-D-thiogalactopyranoside; LB, Luria-Bertani broth; MS, mass spectrometry; MTT, thiazolyl blue tetrazolium bromide; SDS/PAGE, sodium dodecyl sulfate/polyacrylamide gel electrophoresis.
} 
tion of Fas by FasL, has been used for many in vitro studies (Cheng et al., 1994; Nagata, 1997; Raoul et al., 1999; Ciesielski-Treska et al., 2001). Commonly used recombinant Fas-Fc is produced in HEK 293 cells to preserve the pattern of N-linked glycosylation at several sites in the Fas molecule. However, expression and purification of the recombinant Fas-Fc in human cells, is expensive and not readily available, thus alternative methods are desirable.

In the present study we describe a design, cloning, and purification methods to produce of a large amount of recombinant extracellular domain of rat Fas in Escherichia coli. SDS/PAGE and mass spectrometry analysis verified the purity of the recombinant protein preparation. We show that the recombinant protein exhibited biological activities: specific binding of soluble Fas ligand and an efficient blockade of FasL-induced cell death of human lymphoid Jurkat cells.

\section{MATERIALS AND METHODS}

Cloning and expression. Total RNA was isolated from rat brain as described (Zawadzka \& Kaminska, 2005) using an RNA isolation kit (Qiagen). cDNA corresponding to the extracellular domain of mature rat Fas (spanning aa 21-176) was synthesized by extension of specific primers with 200 units of SuperScript II reverse transcriptase (Gibco BRL) and dNTP mix (final concentration 1 $\mu \mathrm{M}$ each). Primer sequences were as follows: $5^{\prime}$ TAGGTCGACAACTGCTTTCTCTTTCTGC-3' and 5'-ACTGGATCCTCTCTTCGTGGCTGGAACCG-3' . The resulting cDNA was amplified by PCR with the second pair of specific primers $(0.15 \mu \mathrm{M}$ each) containing restriction sites (underlined) for NdeI 5'-CTGTCGACCATGCATATGCAAGGGACTGATAGC-3' and for BamHI 5'-GATGGATCCTATCATATCAGCAGCCAAAGGAGC-3'. The resulting PCR product was digested with the restriction enzymes, purified using spun columns, cloned into pET28a $(+)$ expression vector (Novagene) and sequenced. The cloning procedure shifted the open reading frame and the transcription termination site, which resulted in an additional stretch of 41 hydrophilic amino acids constituting a "solubilization enhancing domain".

Escherichia coli (Rosetta strain DE3, Novagen) were transformed by electroporation and 200 $\mathrm{mL}$ of LB medium supplemented with $30 \mu \mathrm{g} / \mathrm{mL}$ of kanamycin was inoculated in a $1: 20$ ratio. The bacteria were cultured at $37^{\circ} \mathrm{C}, 150$ r.p.m. until $\mathrm{OD}_{600}$ reached 0.6. FasL interfering protein expression was induced by $1 \mathrm{mM}$ isopropyl- $\beta$-D-thiogalactopyranoside (IPTG). After a $3 \mathrm{~h}$ incubation bacteria were collected by centrifugation.
Isolation of inclusion bodies and protein purification. Cell pellet was suspended in $10 \mathrm{~mL}$ of cold $50 \mathrm{mM}$ Tris $/ \mathrm{HCl}$ buffer $(\mathrm{pH}$ 8.0) containing $10 \mathrm{mM} \beta$-mercaptoethanol, $1 \mathrm{mM}$ EDTA and $100000 \mathrm{U} / \mathrm{mL}$ lysozyme, and kept for $30 \mathrm{~min}$ on ice. Genomic DNA was fragmented by sonication for $5 \times 20 \mathrm{~s}$ (Digital Sonifier 250, Branson). Crude extract was centrifuged at $14500 \times \mathrm{g}, 20 \mathrm{~min}$ at $4^{\circ} \mathrm{C}$. Pellet was dissolved at room temperature in 100 $\mathrm{mM}$ phosphate buffer, $\mathrm{pH} 7.5$, supplemented with $8 \mathrm{M}$ urea. Denatured preparation was centrifuged at $14500 \times \mathrm{g}, 20 \mathrm{~min}, 4^{\circ} \mathrm{C}$ and the supernatant was subjected to IMAC chromatography using $1.25 \mathrm{~mL}$ of a nickel resin (His-Select, Sigma). The column was equilibrated with $100 \mathrm{mM}$ phosphate buffer, $\mathrm{pH}$ 7.5, containing $8 \mathrm{M}$ urea. Proteins were eluted in a linear gradient of imidazole $(0-250 \mathrm{mM})$ and 33 fractions of $2 \mathrm{~mL}$ were collected. Fractions characterized by the highest amount of protein $\left(\mathrm{A}_{280}>0.5\right)$ were pooled.

Renaturation, fluorometric assay and mass spectrometry analysis. The FIP preparation was diluted to a concentration of $70 \mu \mathrm{g} / \mathrm{mL}$, and subsequently dialyzed overnight at $4^{\circ} \mathrm{C}$ against $50 \mathrm{mM}$ phosphate buffer, $\mathrm{pH} 7.5$, containing $1 \mathrm{mM}$ EDTA and $10 \mathrm{mM} \beta$-mercaptoethanol. The obtained preparation was dialyzed against phosphate-buffered saline (PBS) under the same conditions. Precipitated proteins were removed by centrifugation at $170 \times g$ (1000 r.p.m. in MPW 370 centrifuge, rotator Nr. 12108), $5 \mathrm{~min}$ at $4^{\circ} \mathrm{C}$.

Fluorometric assay was performed using Fluorog 2. Tryptophan fluorescence was excited at $295 \mathrm{~nm}$ and fluorescence spectra were measured in the range of $315-450 \mathrm{~nm}$.

Protein samples were analyzed by liquid chromatography-electrospray mass spectrometry with collision fragmentation using an Electrospray Ionization-Quadrupole-Time Of Flight (ESI-QUADTOF) instrument (Micromass, Manchester, UK). The NCBInr database was searched with the obtained results using the Mascot software. Semitrypsin digestion with a maximum of one missed enzymatic cleavage, Cys and Lys carbamidomethyl and Met oxidation were covered. One microgram of final preparation was visualized on polyacrylamide gel by a silver staining as described elsewhere (Blum et al., 1987).

Native polyacrylamide gel electrophoresis. Separation gel contained 12\% glycerol, $50 \mathrm{mM}$ Tris/ glycine buffer, $\mathrm{pH} 9.1,7.5 \%$ polyacrylamide/bisacrylamide (29:1), $600 \mu \mathrm{g} / \mathrm{mL}$ ammonium persulfate and $0.06 \%$ TEMED. Stacking gel contained $12 \%$ glycerol, $50 \mathrm{mM}$ Tris/glycine buffer, $\mathrm{pH}$ 9.1, $4 \%$ polyacrylamide/bisacrylamide $(29: 1), 800 \mu \mathrm{g} / \mathrm{mL}$ ammonium persulfate and $0.12 \%$ TEMED. The protein preparation was mixed with glycerol $(1: 10)$ and loaded 
onto the gel. Electrophoresis was performed at 12.5 $\mathrm{V} / \mathrm{cm}, 4^{\circ} \mathrm{C}$.

Western blotting. Native polyacrylamide gel electrophoresis was followed by electrotransfer $\left(400 \mathrm{~mA}, 90 \mathrm{~min}, 4^{\circ} \mathrm{C}\right)$ onto nitrocellulose membrane (Hybond, Amersham Biosciences). The membrane was incubated with anti-His-tag monoclonal antibody (Sigma) and subsequently with anti-mouse antibody linked to horseradish peroxidase. Immunocomplexes were visualized using the enhanced chemiluminescence detection system (ECL, Amersham Biosciences).

Cell viability (MTT metabolism assay). Jurkat cells were cultured in RPMI-1640 medium supplemented with $10 \%$ fetal bovine serum and antibiotics (50 units $/ \mathrm{mL}$ penicillin, $50 \mu \mathrm{g} / \mathrm{mL}$ streptomycin). Cells were sieved at a concentration of $6 \times 10^{4}$ cells/mL in 24 -well culture plates in a total volume of $0.5 \mathrm{~mL}$. Cells grew in a humidified atmosphere of $\mathrm{CO}_{2} /$ air $(5 \% / 95 \%)$ at $37^{\circ} \mathrm{C}$. Twenty-four hours after plating the cells were treated with $400 \mathrm{ng}$ of recombinant rat FasL (R\&D) alone or in combination with $1.6 \mu \mathrm{g} / \mathrm{mL}$ of FIP or PBS. Twenty-four hours after the addition of FasL, MTT stock solution was added to a final concentration of $0.5 \mathrm{mg} / \mathrm{mL}$. After $2 \mathrm{~h}$ of incubation at $37^{\circ} \mathrm{C}$ formazan crystals formed in actively metabolizing cells were dissolved in lysis buffer containing $20 \%$ SDS, $2.5 \% \mathrm{HCl}, 2.5 \%$ acetic acid and $50 \%$ DMF. Absorbance was measured at $570 \mathrm{~nm}$ using a scanning multi-well spectrophotometer. All measurements were carried out in triplicates.

\section{RESULTS}

Purification of FasL interfering protein under denaturing conditions followed by refolding

Recombinant FIP protein was produced in E. coli Rosetta strain DE3 transformed with pET28a(+) plasmid coding for the extracellular domain of Fas receptor ( $\Delta$ Fas) fused to a histidine tag and a solubilization enhancing domain (Fig. 1A and B). The expression of FIP was induced by addition of $1 \mathrm{mM}$ IPTG for $3 \mathrm{~h}$ at $37^{\circ} \mathrm{C}$. Bacterial cells were lyzed by lysozyme digestion and sonication, and the extracts were separated into soluble and insoluble fractions by centrifugation. When aliquots of those fractions were analyzed by staining of SDS/PAGE gels with Coomassie Brilliant Blue (Fig. 1D, lane 3 and 4$)$, a prominent protein band $(25 \mathrm{kDa})$ was found in the insoluble fraction of the crude extract (Fig. 1D, lane 4).

Recombinant FIP was solubilized from inclusion bodies with $8 \mathrm{M}$ urea, purified on an affinity columns and refolded by dialysis. Typically, $40 \mathrm{mg}$ of total solubilized proteins from $200 \mathrm{~mL}$ of induced E. coli culture was loaded onto a $\mathrm{Ni}^{2+}$-column and the bound proteins were eluted with $250 \mathrm{mM}$ imidazole. The presence of recombinant protein in elution fractions was confirmed by immunoblotting with specific anti-6xHis antibody (Fig. 3). This step gave a relatively good result ending up with a highly purified preparation (Fig. 1D, lane 6). At this stage the determined yield was $14 \mathrm{mg}$ of recombinant protein per $1 \mathrm{~L}$ of culture. The molecular mass of the recombinant FIP protein was about $25 \mathrm{kDa}$, as determined by SDS/PAGE, in agreement with the predicted molecular mass.

The purified preparation was dialyzed overnight against phosphate buffer supplemented with EDTA (to avoid protein aggregation) and $\beta$-mercaptoethanol. The removal of urea resulted in protein precipitation. The final protein concentration was $20 \mu \mathrm{g} / \mathrm{mL}$. The total yield was $4 \mathrm{mg}$ of native protein per $1 \mathrm{~L}$ of culture.

A fluorometric assay based on tryptophan spectra shifts, was performed in order to evaluate protein folding after renaturation. The spectrum of tryptophan fluorescence in the preparation subjected to dialysis had a maximum at $347 \mathrm{~nm}$, shifted by 20 $\mathrm{nm}$ as compared to the preparation containing $8 \mathrm{M}$ urea (with a maximum at $367 \mathrm{~nm}$ ), showing conformational changes placing tryptophan residues into a more hydrophobic environment. In contrast, FIP preparation rapidly diluted 100 -fold in dialyzing buffer had a fluorescence with a maximum at $375 \mathrm{~nm}$ (Fig. 2) and exhibited no biological activity (not shown).

In order to estimate the protein purity, $1 \mu \mathrm{g}$ of the final preparation was run on SDS/PAGE followed by silver staining. FIP constituted about $80 \%$ of total proteins (Fig. 1E). The remaining 20\% was represented by two major additional bands with molecular masses of about $18.8 \mathrm{kDa}$ and $15.9 \mathrm{kDa}$. The final preparation was subjected to MS/MS analysis using an Electrospray Ionization-Quadrupole-Time Of Flight (ESI-QUAD-TOF) instrument. The obtained results identified additional proteins as E. coli glutamate synthase (GI 551808, corresponding to $18.8 \mathrm{kDa}$ ), $\beta$-galactosidase $\alpha$ peptide (GI 3559832, corresponding to $15.9 \mathrm{kDa}$ ) and transposition regulator protein (GI 10955466, corresponding to 50 $\mathrm{kDa}$ ). This analysis confirmed a lack of potentially toxic bacteria-derived proteins which could affect mammalian cells.

\section{Evaluation of biological activity of recombinant FIP}

To detect direct protein-protein interactions, FIP and recombinant rat FasL, as well as appropriate controls, were incubated overnight at $4^{\circ} \mathrm{C}$. Subsequently, the preparations were run on a native 


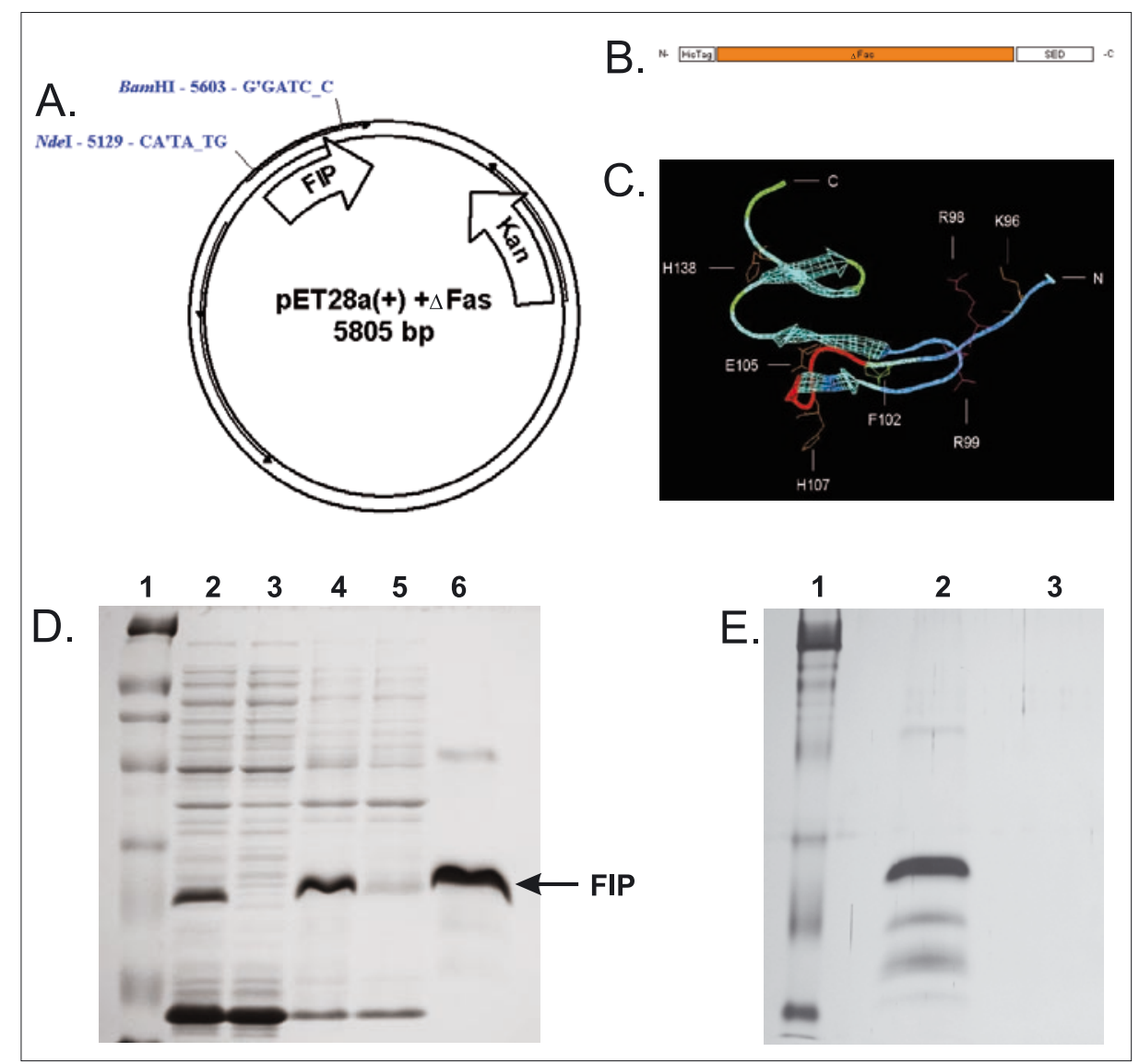

Figure 1. Schematic representation of plasmid encoding FIP, predicted FIP tertiary structure and SDS/PAGE of affinity-purified recombinant FIP.

A-B. A schematic view of the construct: cDNA encoding a truncated extracellular domain of rat Fas was cloned into pET28a $(+)$ vector (A) and the expressed recombinant protein (FIP) consisting of Histidine Tag (HisTag), extracellular domain of rat Fas receptor $(\Delta \mathrm{Fas})$ and a solubilization enhancing domain (SED) (B). C. Tertiary structure of FIP was predicted using First Approach mode at Automated Comparative Protein Modelling Server (Swiss-Model) by means of the following PDB templates: 1tnrR, 1ncfB, 1extA, 1ft4B. Amino acids potentially involved in binding of FasL are indicated. D. Polyacrylamide gel stained with Coomassie Brilliant Blue. Lane description: 1. Molecular mass standards (Sigma C1992-1VL); 2. E. coli crude extract; 3. Supernatant collected after crude extract centrifugation; 4 . Pellet collected after crude extract centrifugation; 5. Flow-through collected during metal affinity chromatography; 6 . Fraction eluted from nickel resin. E. One $\mu \mathrm{g}$ of FIP final preparation was run on SDS/PAGE and silver stained. Lanes: 1. Molecular weight standards (SIGMA C1992-1VL), 2. FIP final preparation, 3. Control buffer.

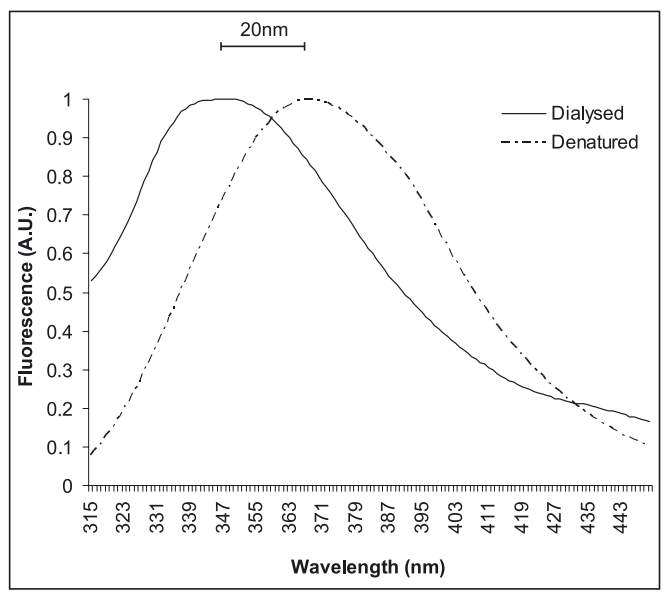

Figure 2. Verification of protein folding after renaturation. Fluorescence spectra of protein preparations: denatured, rapidly diluted in renaturation buffer, and subjected to renaturation process. The protein concentration was $20 \mu \mathrm{g} / \mathrm{ml}$. polyacrylamide gel at $4^{\circ} \mathrm{C}$ in order to preserve the preformed FIP-FasL complexes. Figure 3A presents the result of a Western blot analysis, which reveals a shift of the band representing FIP-FasL complexes as compared to FasL alone.

The biological, cytotoxic activity of recombinant rat FasL can be measured by evaluating its ability to induce apoptosis of Jurkat lymphoid cells. Therefore, to evaluate recombinant FIP activity in a biological system, Jurkat cells were cultured in the presence of FasL alone or together with FIP in a molar ratio of $1: 4$ (FasL/FIP). The cell viability, determined by the MTT metabolism test, was evaluated after $24 \mathrm{~h}$. The obtained results show an approx. $30 \%$ decrease in cell viability in cultures treated with FasL alone, whereas the viability of cells treated with FasL/FIP was unaffected and remained at the 


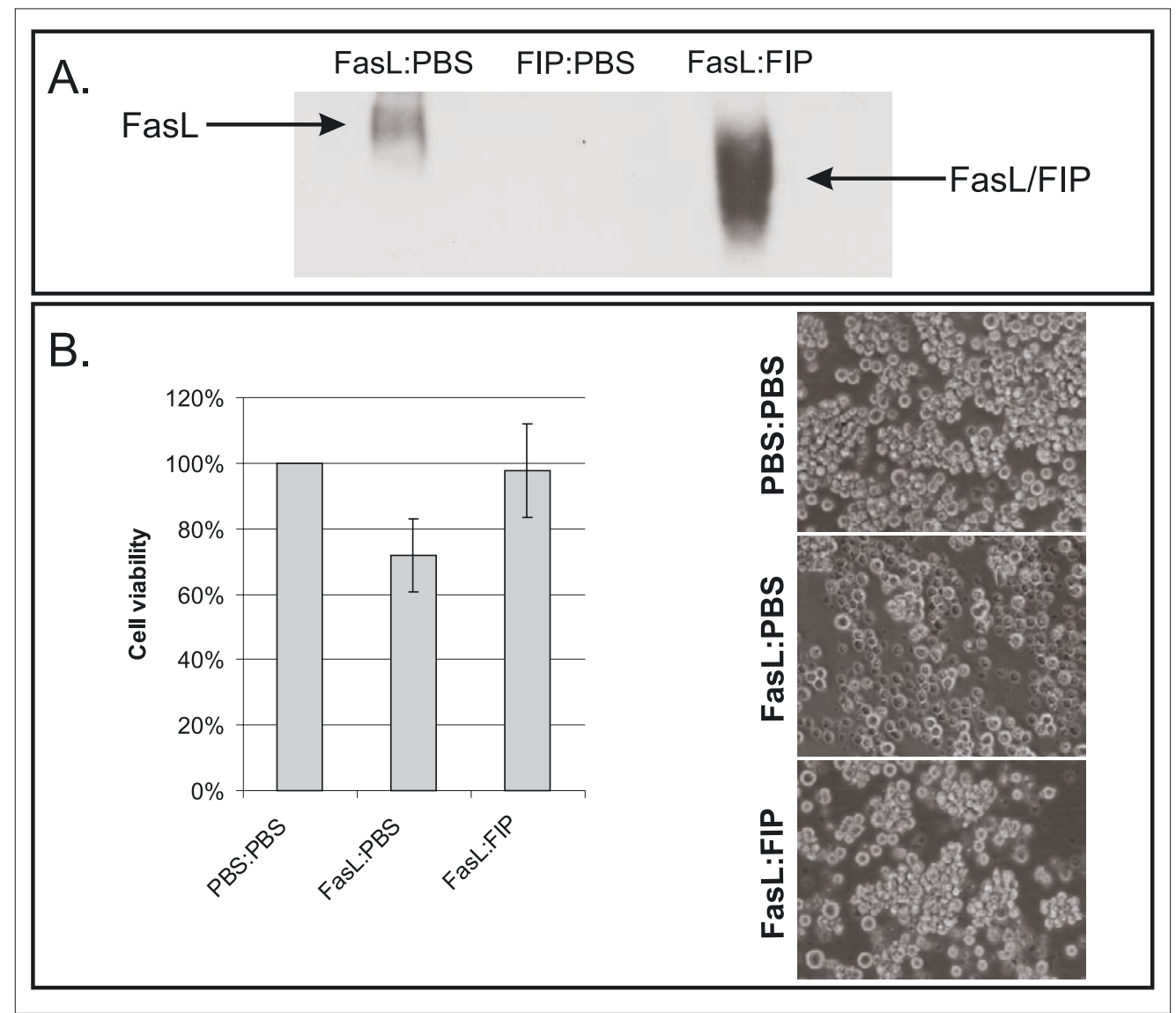

Figure 3. Evaluation of FIP biological activity.

A. Recombinant rat FasL or FIP alone or a combination of FasL with FIP in a molar ratio of $1: 1$ were incubated overnight. Subsequently, preparations were run on native PAGE, transferred onto a nitrocellulose membrane and probed with an anti-His-tag monoclonal antibody. B. Jurkat lymphoid cells were treated with recombinant rat FasL or with FasL and FIP in a molar ratio of $1: 4$. Cell viability was measured by MTT metabolism test $24 \mathrm{~h}$ after treatment (left panel). Right panel shows phase-contrast microscopy pictures of treated cells.

level of untreated cells (Fig. 3B). Phase-contrast microscopy shows a reduction of cell proliferation and colony formation in FasL-treated Jurkat cell cultures, while co-treatment with recombinant FIP blocked those changes.

\section{DISCUSSION}

A major cell surface receptor involved in the induction of apoptosis is Fas that is activated by binding Fas ligand (Curtin \& Cotter, 2003). Fas-Fc, which contains the extracellular domain of recombinant human Fas (aa 1-154) fused to the Fc domain of human IgG1, antagonizes the activation of Fas by FasL. Fas-Fc peptide has been extensively used for many in vitro studies, mostly to verify if stimulusinduced apoptosis was mediated through Fas-FasL interactions (Cheng et al., 1994; Nagata, 1997; Raoul et al., 1999). Commonly used recombinant Fas-Fc is produced in human HEK 293 cells to preserve the pattern of N-linked glycosylation at several sites in the Fas molecule. Although commercially available, Fas-Fc is very expensive and used mostly for cell culture studies. It is not suitable for studies in rat models of disease.

In the present study we demonstrate the purification of the extracellular domain of rat Fas protein, called FIP (FasL interfering protein), expressed as inclusion bodies in E. coli. FIP was solubilized from inclusion bodies through denaturation, affinity chromatography and refolding. The final preparation was highly purified, $80 \%$ homogenous and MS/MS analysis revealed the presence of only traces of three bacterial proteins. The fluorometric assay based on tryptophan spectra shifts confirmed the protein folding after renaturation.

Domain deletion and mutational analysis of amino-acid residues in the Fas extracellular domain demonstrated critical residues in two domains that correspond to positions critical for ligand binding (Itoh \& Nagata, 1993; Starling et al., 1997; 1998). Two 
amino acids, R86 and R87 in domain 2, are critical for the ability of Fas to interact with its ligand (Starling et al., 1997).

Direct interactions between recombinant FIP and recombinant rat FasL protein were demonstrated by detection with anti-His-tag antibody preformed complexes of FIP-FasL separated by native gel electrophoresis. Purified FIP exhibited a biological activity and blocked FasL-induced apoptosis of Jurkat lymphoid cells. Recombinant rat FIP used in this study efficiently blocked the decrease in cell viability of human lymphoid cells induced by recombinant rat FasL.

It has been widely accepted that preservation of N-linked glycosylation pattern in the Fas molecule is necessary for its biological activity and secretion, thus it has to be expressed in mammalian cells (Li et al., 2007). Our studies demonstrate that the extracellular domain of Fas expressed in E. coli is biologically active and retains the ability to interfere with FasL signalling. Although the recombinant FIP emerged as inclusion bodies, it could efficiently be solubilized with $8 \mathrm{M}$ urea and purified to almost electrophoretic homogeneity by a single-step immobilized metal ion $\left(\mathrm{Ni}^{2+}\right)$ affinity chromatography and refolded by simple dialysis. This efficient procedure of FIP purification and renaturation may be useful for production of large quantities of this therapeutically important protein. It offers a simple alternative to the costly procedure of recombinant protein purification from human cells.

\section{Acknowledgements}

This work was supported by a grant N301 394533 from The Ministry of Science and Higher Education.

\section{REFERENCES}

Barnhart BC, Legembre P, Pietras E, Bubici E, Franzoso G, Peter ME (2004) CD95 ligand induces motility and invasiveness of apoptosis-resistant tumor cells. EMBO J 23: 3175-3185.

Blum H, Bayer H, Gross HJ (1987) Improved silver staining of plant proteins, RNA and DNA in polyacrylamide gels. Electrophoresis 8: 93-99.

Cheng J, Zhou T, Liu C, Shapiro JP, Brauer MJ, Kiefer MC, Barr PJ, Mountz DJ (1994) Protection from Fas-mediated apoptosis by a soluble form of the Fas molecule. Science 263: 1759-1762.

Choi C, Benveniste EN (2004) Fas ligand/Fas system in the brain: regulator of immune and apoptotic responses. Brain Res Brain Res Rev 44: 65-81.

Ciesielski-Treska J, Ulrich G, Taupenot L, Chasserot-Golaz S, Zwiller J, Revel MO, Aunis D, Bader MF (2001)
Mechanisms underlying neuronal death induced by chromogranin A-activated microglia. J Biol Chem 276: 13113-13120.

Curtin JF, Cotter TG (2003) Live and let die: regulatory mechanisms in Fas-mediated apoptosis. Cell Signal 15: 983-992.

Ethell DW, Buhler LA (2003) Fas ligand-mediated apoptosis in degenerative disorders of the brain. J Clin Immunol 23: 363-370.

Green DR (2003) Introduction: apoptosis in the development and function of the immune system. Semin Immunol 15: 121-123.

Guex N, Peitsch MC (1997) SWISS-MODEL and the SwissPdbViewer: An environment for comparative protein modeling. Electrophoresis 18: 2714-2723.

Itoh N, Nagata S (1993) A novel protein domain required for apoptosis. Mutational analysis of human Fas antigen. J Biol Chem 268: 10932-10937.

Krammer PH (2000) CD95's deadly mission in the immune system. Nature 407: 789-795.

Li Y, Yang X, Nguyen AH, Brockhausen I (2007) Requirement of N-glycosylation for the secretion of recombinant extracellular domain of human Fas in HeLa cells. Int J Biochem Cell Biol 39: 1625-1636.

Nagata S (1997) Apoptosis by death factor. Cell 88: 355365.

Peitsch MC (1995) Protein modeling by E-mail. Bio/Technology 13: 658-660.

Raoul C, Henderson CE, Pettmann B (1999) Programmed cell death of embryonic motoneurons triggered through the Fas death receptor. J. Cell Biol 29: 1049-1061.

Ryan AE, Shanahan F, O'Connell J, Houston AM (2005) Addressing the "Fas counterattack" controversy: blocking Fas ligand expression suppresses tumor immune evasion of colon cancer in vivo. Cancer Res 65: 98179823.

Scaffidi C, Fulda S, Srinivasan A, Friesen C, Li F, Tomaselli KJ, Debatin KM, Krammer PH, Peter ME (1998) Two CD95 (APO-1/Fas) signaling pathways. EMBO J 17: $1675-1687$.

Schwede T, Kopp J, Guex N, Peitsch MC (2003) SWISSMODEL: an automated protein homology-modeling server. Nucleic Acids Res 31: 3381-3385.

Smith CA, Farrah T, Goodwin RG (1994) The TNF receptor superfamily of cellular and viral proteins: activation, costimulation, and death. Cell 76: 959-962.

Starling GC, Bajorath J, Emswiler J, Ledbetter JA, Aruffo A, Kiener PA (1997) Identification of amino acid residues important for ligand binding to Fas. J Exp Med 185: 1487-1492.

Starling GC, Kiener PA, Aruffo A, Bajorath J (1998) Analysis of the ligand binding site in Fas (CD95) by sitedirected mutagenesis and comparison with TNFR and CD40. Biochemistry 37: 3723-3726.

Wajant H, Pfizenmaier K, Scheurich P (2003) Non-apoptotic Fas signaling. Cytokine Growth Factor Rev 14: 53-66.

Zawadzka M, Kaminska B (2005) A novel mechanism of FK506-mediated neuroprotection: downregulation of cytokine expression in glial cells. Glia 49: 36-51. 